\title{
ON THE BREIMAN CONJECTURE
}

\author{
PÉTER KEVEI AND DAVID M. MASON
}

(Communicated by Mark M. Meerschaert)

\begin{abstract}
Let $Y_{1}, Y_{2}, \ldots$ be positive, nondegenerate, i.i.d. $G$ random variables, and independently let $X_{1}, X_{2}, \ldots$ be i.i.d. $F$ random variables. In this note we show that for $F \in \mathcal{F}$ in a specified class of distributions $\mathcal{F}$, whenever $\sum X_{i} Y_{i} / \sum Y_{i}$ converges in distribution to a nondegenerate limit then $\mathrm{G}$ necessarily belongs to the domain of attraction of a stable law with index less than 1. The class $\mathcal{F}$ contains those nondegenerate $X$ with a finite second moment and those $X$ in the domain of attraction of a stable law with index $1<\alpha<2$.
\end{abstract}

\section{INTRODUCTION AND RESULtS}

Let $Y, Y_{1}, \ldots$ be positive, nondegenerate, i.i.d. random variables with distribution function $[\mathrm{df}] G$, and independently let $X, X_{1}, \ldots$ be i.i.d. nondegenerate random variables with df $F$. Let $\phi_{X}$ denote the characteristic function [cf] of $X$. We shall use the notation $Y \in D(\beta)$ to mean that $Y$ is in the domain of attraction of a stable law of index $0<\beta<1$, and $Y \in D(0)$ will denote that $1-G$ is slowly varying at infinity. Furthermore, $\mathcal{R} \mathcal{V}_{\infty}(\rho)$ will signify the class of positive measurable functions regularly varying at infinity with index $\rho$, and $\mathcal{R} \mathcal{V}_{0}(\rho)$ the class of positive measurable functions regularly varying at zero with index $\rho$. In particular, using this notation $Y \in D(\beta)$, with $0 \leq \beta<1$, if and only if $\bar{G}:=$ $1-G \in \mathcal{R} \mathcal{V}_{\infty}(-\beta)$.

For each integer $n \geq 1$ set

$$
T_{n}=\sum_{i=1}^{n} X_{i} Y_{i} / \sum_{i=1}^{n} Y_{i} .
$$

Notice that $\mathbb{E}|X|<\infty$ implies that $T_{n}$ is stochastically bounded. Theorem 4 of Breiman [2] says that $T_{n}$ converges in distribution along the full sequence $\{n\}$ for $e v$ ery $X$ with finite expectation, and with at least one limit law being nondegenerate, if and only if

$$
Y \in D(\beta) \text {, with } 0 \leq \beta<1 \text {. }
$$

Let $\mathcal{X}$ denote the class of nondegenerate random variables $X$ with $\mathbb{E}|X|<\infty$ and let $\mathcal{X}_{0}$ denote those $X \in \mathcal{X}$ such that $\mathbb{E} X=0$. At the end of his paper Breiman conjectured that if for some $X \in \mathcal{X}, T_{n}$ converges in distribution to some nondegenerate random variable $T$, written

$$
T_{n} \rightarrow_{d} T \text {, as } n \rightarrow \infty \text {, with } T \text { nondegenerate, then (2) holds. }
$$

Received by the editors August 6, 2015 and, in revised form, October 28, 2015.

2010 Mathematics Subject Classification. Primary 60F05.

The research of the first author was funded by a postdoctoral fellowship of the Alexander von Humboldt Foundation. 
By Proposition 2 (in the case $\beta=0$ ) and Theorem 3 (in the case $0<\beta<1$ ) of [2], for any $X \in \mathcal{X},(2)$ implies (3), in which case $T$, in the case $0<\beta<1$, has a distribution related to the arcsine law. Using this fact, we see that his conjecture can be restated to be: for any $X \in \mathcal{X},(2)$ is equivalent to (3).

It has proved to be surprisingly challenging to resolve. Mason and Zinn [8] partially verified Breiman's conjecture. They established that whenever $X$ is nondegenerate and satisfies $\mathbb{E}|X|^{p}<\infty$ for some $p>2$, then (2) is equivalent to (3). In this note we further extend this result.

Theorem 1. Assume that for some $X \in \mathcal{X}_{0}, 1<\alpha \leq 2$, positive slowly varying function $L$ at zero and $c>0$,

$$
\frac{-\log \left(\mathfrak{R e} \phi_{X}(t)\right)}{|t|^{\alpha} L(|t|)} \rightarrow c, \text { as } t \rightarrow 0 .
$$

Whenever (3) holds, then $Y \in D(\beta)$ for some $\beta \in[0,1)$.

Let $\mathcal{F}$ denote the class of random variables that satisfy the conditions of the theorem. Applying our theorem in combination with Proposition 2 and Theorem 3 of [2] we get the following corollary.

Corollary 1. Whenever $X-\mathbb{E} X \in \mathcal{F}$, (2) is equivalent to (3).

Remark 1. It can be inferred from Theorem 8.1.10 of Bingham et al. 1] (see also Theorems 1 and 5 of Pitman [9]) that for $X \in \mathcal{X}_{0}$, (4) holds for some $1<\alpha<2$, positive slowly varying function $L$ at zero and $c>0$ if and only if $X$ satisfies $\mathbb{P}\{|X|>x\} \sim L(1 / x) x^{-\alpha} c \Gamma(\alpha) \frac{2}{\pi} \sin \left(\frac{\pi \alpha}{2}\right)$. Note that a random variable $X \in \mathcal{X}_{0}$ in the domain of attraction of a stable law of index $1<\alpha<2$ satisfies (44). For $\alpha=2$ there is no simple condition equivalent to (4). By Theorem 5 of Pitman [9], for $\alpha=2$ condition (4) implies that

$$
\frac{1-\mathfrak{k e} \phi_{X}(t)}{t^{2}} \sim \int_{0}^{t^{-1}} u \mathbb{P}\{|X|>u\} \mathrm{d} u, \quad \text { as } t \downarrow 0 .
$$

Also a random variable $X \in \mathcal{X}_{0}$ with variance $0<\sigma^{2}<\infty$ fulfills (4) with $\alpha=2$, $L=1$ and $c=\sigma^{2} / 2$. Theorem 3 in [9] states that $\mathbb{P}\{|X|>x\} \in \mathcal{R} \mathcal{V}_{\infty}(-2)$ implies (5), from which, combined with Proposition 1.5.9a of [1, condition (4) follows.

Remark 2. Consult Kevei and Mason [7] for a fairly exhaustive study of the asymptotic distributions of $T_{n}$ along subsequences, along with revelations of their unexpected properties.

The theorem follows from the two propositions below. First we need more notation. For any $\alpha \in(1,2]$, define for $n \geq 1$

$$
S_{n}(\alpha)=\frac{\sum_{i=1}^{n} Y_{i}^{\alpha}}{\left(\sum_{i=1}^{n} Y_{i}\right)^{\alpha}} .
$$

Proposition 1. Assume that the assumptions of the theorem hold. Then for some $0<\gamma \leq 1$,

$$
\mathbb{E} S_{n}(\alpha) \rightarrow \gamma, \text { as } n \rightarrow \infty .
$$

The next proposition is interesting in its own right. It is an extension of Theorem 5.3 by Fuchs et al. [4, where $\alpha=2$ (see also Proposition 3 of [ $]$ ). 
Proposition 2. If (7) holds with some $\gamma \in(0,1]$, then $Y \in D(\beta)$, for some $\beta \in[0,1)$, where $-\beta \in(-1,0]$ is the unique solution of

$$
\operatorname{Beta}(\alpha-1,1-\beta)=\frac{\Gamma(\alpha-1) \Gamma(1-\beta)}{\Gamma(\alpha-\beta)}=\frac{1}{\gamma(\alpha-1)} .
$$

In particular, $Y \in D(0)$ for $\gamma=1$.

Conversely, if $Y \in D(\beta), 0 \leq \beta<1$, then (7) holds with

$$
\gamma=\frac{\Gamma(\alpha-\beta)}{\Gamma(\alpha) \Gamma(1-\beta)}=\frac{1}{(\alpha-1) \operatorname{Beta}(\alpha-1,1-\beta)} .
$$

\section{Proofs}

Set for each $n \geq 1, R_{i}=Y_{i} / \sum_{l=1}^{n} Y_{l}$, for $i=1, \ldots, n$. For notational ease we drop the dependence of $R_{i}$ on $n \geq 1$. Consider the sequence of strictly decreasing continuous functions $\left\{\varphi_{n}\right\}_{n \geq 1}$ on $[1, \infty)$ defined by $\varphi_{n}(y)=\mathbb{E}\left(\sum_{i=1}^{n} R_{i}^{y}\right), y \in$ $[1, \infty)$. Note that each function $\varphi_{n}$ satisfies $\varphi_{n}(1)=1$. By a diagonal selection procedure for each subsequence of $\{n\}_{n>1}$ there is a further subsequence $\left\{n_{k}\right\}_{k \geq 1}$ and a right continuous nonincreasing function $\psi$ such that $\varphi_{n_{k}}$ converges to $\psi$ at each continuity point of $\psi$.

Lemma 1. Each such function $\psi$ is continuous on $(1, \infty)$.

Proof. Choose any subsequence $\left\{n_{k}\right\}_{k \geq 1}$ and a right continuous nonincreasing function $\psi$ such that $\varphi_{n_{k}}$ converges to $\psi$ at each continuity point of $\psi$ in $(1, \infty)$. Select any $x>1$ and continuity points $x_{1}, x_{2} \in(1, \infty)$ of $\psi$ such that $1<x_{1}<x<x_{2}<$ $\infty$. Set $\rho_{1}=x_{1}-1$ and $\rho_{2}=x_{2}-1$. Since $\rho_{2} / \rho_{1}>1$ we get by Hölder's inequality

$$
\sum_{i=1}^{n_{k}} R_{i}^{x_{1}}=\sum_{i=1}^{n_{k}} R_{i}^{\rho_{1}} R_{i} \leq\left(\sum_{i=1}^{n_{k}} R_{i}^{\rho_{2}} R_{i}\right)^{\rho_{1} / \rho_{2}}=\left(\sum_{i=1}^{n_{k}} R_{i}^{x_{2}}\right)^{\rho_{1} / \rho_{2}} .
$$

Taking expectations and using Jensen's inequality we get $\varphi_{n_{k}}\left(x_{1}\right) \leq\left(\varphi_{n_{k}}\left(x_{2}\right)\right)^{\rho_{1} / \rho_{2}}$. Letting $n_{k} \rightarrow \infty$, we have $\psi\left(x_{1}\right) \leq\left(\psi\left(x_{2}\right)\right)^{\rho_{1} / \rho_{2}}$. Since $x_{1}<x$ and $x_{2}>x$ can be chosen arbitrarily close to $x$ we conclude by right continuity of $\psi$ at $x$ that $\psi(x-)=\psi(x+)=\psi(x)$.

Proof of Proposition 1. For a complex $z$, we use the notation for the principal branch of the $\operatorname{logarithm}, \log (z)=\log |z|+\imath \arg z$, where $-\pi<\arg z \leq \pi$, i.e. $z=|z| \exp (\imath \arg z)$. We see that for all $t$

$$
\begin{aligned}
\mathbb{E} \exp \left(\imath t T_{n}\right) & =\mathbb{E}\left(\prod_{j=1}^{n} \phi_{X}\left(t R_{j}\right)\right) \\
& =\mathbb{E}\left(\prod_{j=1}^{n} \exp \left(\log \phi_{X}\left(t R_{j}\right)\right)\right) .
\end{aligned}
$$

Since $\mathbb{E} X=0$ we have $\mathfrak{R e} \phi_{X}(u)=1-o_{+}(u)$, where $o_{+}(u) \geq 0$ and $o_{+}(u) / u \rightarrow 0$ as $u \rightarrow 0$, and $\mathfrak{I m} \phi_{X}(u)=o(u)$. This when combined with

$$
(\arctan \theta)^{\prime}=\frac{1}{1+\theta^{2}}
$$


gives as $u \rightarrow 0$,

$$
\arg \phi_{X}(u)=\arctan \left(\frac{\mathfrak{I m} \phi_{X}(u)}{\mathfrak{R e} \phi_{X}(u)}\right)=o(u) .
$$

Note that for all $|u|>0$ sufficiently small so that $\mathfrak{R e} \phi_{X}(u)>0$,

$\log \phi_{X}(u)=\log \left(\mathfrak{R e} \phi_{X}(u)+\imath \mathfrak{I m} \phi_{X}(u)\right)=\log \mathfrak{R e} \phi_{X}(u)+\log \left(1+\imath \frac{\mathfrak{I m} \phi_{X}(u)}{\mathfrak{R e} \phi_{X}(u)}\right)$,

where for the second term

$$
\mathfrak{R e} \log \left(1+\imath \frac{\mathfrak{I m} \phi_{X}(u)}{\mathfrak{R e} \phi_{X}(u)}\right)=\frac{1}{2}\left(\frac{\mathfrak{I m} \phi_{X}(u)}{\mathfrak{R e} \phi_{X}(u)}\right)^{2}(1+o(u)), \text { as } u \rightarrow 0 .
$$

Thus for every $\varepsilon>0$ for all $|t|>0$ sufficiently small and independent of $n \geq 1$ and $R_{1}, \ldots, R_{n}$,

$1-\varepsilon^{2} t^{2} \leq \cos (\varepsilon t) \leq \mathfrak{R e}\left(\exp \left\{\sum_{j=1}^{n} \log \left(1+\imath \frac{\mathfrak{I m} \phi_{X}\left(t R_{j}\right)}{\mathfrak{R e} \phi_{X}\left(t R_{j}\right)}\right)\right\}\right) \leq e^{2^{-1} \varepsilon t^{2}} \leq 1+\varepsilon t^{2}$.

Thus we obtain

$$
\begin{aligned}
\mathbb{E} \exp \left\{\sum_{j=1}^{n} \log \mathfrak{R e} \phi_{X}\left(t R_{j}\right)\right\}\left(1-\varepsilon^{2} t^{2}\right) & \leq \mathbb{E}\left(\mathfrak{R e} \exp \left(\imath t T_{n}\right)\right) \\
& =\mathfrak{R e} \mathbb{E} \exp \left(\imath t T_{n}\right) \\
& \leq \mathbb{E} \exp \left\{\sum_{j=1}^{n} \log \mathfrak{R e} \phi_{X}\left(t R_{j}\right)\right\}\left(1+\varepsilon t^{2}\right) .
\end{aligned}
$$

We shall show (44) implies that (7) holds for some $0<\gamma \leq 1$. Now using (44) we get for any $0<\delta<c$ and all $|t|$ small enough independent of $n \geq 1$,

$$
\begin{aligned}
& -\varepsilon t^{2}+\log \mathbb{E} \exp \left(-(c+\delta)|t|^{\alpha}\left(\sum_{i=1}^{n} R_{i}^{\alpha} L\left(|t| R_{i}\right)\right)\right) \leq \log \left(\mathfrak{R e} \mathbb{E} \exp \left(\imath t T_{n}\right)\right) \\
& \leq \varepsilon t^{2}+\log \mathbb{E} \exp \left(-(c-\delta)|t|^{\alpha}\left(\sum_{i=1}^{n} R_{i}^{\alpha} L\left(|t| R_{i}\right)\right)\right) .
\end{aligned}
$$

Next since $\log s /(1-s) \rightarrow-1$ as $s \nearrow 1$, for all $|t|$ small enough independent of $n \geq 1$ and $R_{1}, \ldots, R_{n}$ (keeping in mind that $\sum_{i=1}^{n} R_{i}=1$ and $1<\alpha \leq 2$ ),

$$
\begin{aligned}
& \log \mathbb{E} \exp \left(-(c+\delta)|t|^{\alpha}\left(\sum_{i=1}^{n} R_{i}^{\alpha} L\left(|t| R_{i}\right)\right)\right) \\
& \geq-\left(1+\frac{\delta}{2}\right) \mathbb{E}\left(1-\exp \left(-(c+\delta)|t|^{\alpha}\left(\sum_{i=1}^{n} R_{i}^{\alpha} L\left(|t| R_{i}\right)\right)\right)\right)
\end{aligned}
$$

and

$$
\begin{aligned}
& \log \mathbb{E} \exp \left(-(c-\delta)|t|^{\alpha}\left(\sum_{i=1}^{n} R_{i}^{\alpha} L\left(|t| R_{i}\right)\right)\right) \\
& \leq-\left(1-\frac{\delta}{2}\right) \mathbb{E}\left(1-\exp \left(-(c-\delta)|t|^{\alpha}\left(\sum_{i=1}^{n} R_{i}^{\alpha} L\left(|t| R_{i}\right)\right)\right)\right) .
\end{aligned}
$$


Further, since $(1-\exp (-y)) / y \rightarrow 1$ as $y \searrow 0$, for all $|t|$ small enough independent of $n \geq 1$,

$$
\begin{aligned}
& -\left(1+\frac{\delta}{2}\right) \mathbb{E}\left(1-\exp \left(-(c+\delta)|t|^{\alpha}\left(\sum_{i=1}^{n} R_{i}^{\alpha} L\left(|t| R_{i}\right)\right)\right)\right) \\
& \geq-(1+\delta)(c+\delta)|t|^{\alpha} \mathbb{E}\left(\sum_{i=1}^{n} R_{i}^{\alpha} L\left(|t| R_{i}\right)\right)
\end{aligned}
$$

and

$$
\begin{aligned}
& -\left(1-\frac{\delta}{2}\right) \mathbb{E}\left(1-\exp \left(-(c-\delta)|t|^{\alpha}\left(\sum_{i=1}^{n} R_{i}^{\alpha} L\left(|t| R_{i}\right)\right)\right)\right) \\
& \leq-(1-\delta)(c-\delta)|t|^{\alpha} \mathbb{E}\left(\sum_{i=1}^{n} R_{i}^{\alpha} L\left(|t| R_{i}\right)\right) .
\end{aligned}
$$

Therefore, for all $|t|$ small enough independent of $n$,

$$
\begin{aligned}
& -\varepsilon t^{2}-(1+\delta)(c+\delta)|t|^{\alpha} \mathbb{E}\left(\sum_{i=1}^{n} R_{i}^{\alpha} L\left(|t| R_{i}\right)\right) \\
& \leq \log \left(\mathfrak{R e} \mathbb{E} \exp \left(\imath t T_{n}\right)\right) \\
& \leq \varepsilon t^{2}-(1-\delta)(c-\delta)|t|^{\alpha} \mathbb{E}\left(\sum_{i=1}^{n} R_{i}^{\alpha} L\left(|t| R_{i}\right)\right) .
\end{aligned}
$$

By the Potter's bound, Theorem 1.5.6 (i) in [1], for all $A>1$ and $1<\alpha_{1}<\alpha<\alpha_{2}$, for all $t>0$ small enough independent of $n \geq 1$,

$$
A^{-1} \sum_{i=1}^{n} R_{i}^{\alpha_{2}} \leq \sum_{i=1}^{n} R_{i}^{\alpha} L\left(|t| R_{i}\right) / L(|t|) \leq A \sum_{i=1}^{n} R_{i}^{\alpha_{1}} .
$$

We see now that for all $n \geq 1$ and $0<4 \varepsilon<c$, appropriate $1<\alpha_{1}<\alpha<\alpha_{2}$ and all $|t|$ small enough independent of $n$,

$$
\begin{aligned}
& -\varepsilon t^{2}-(1+\varepsilon)(c+2 \varepsilon)|t|^{\alpha} L(|t|) \mathbb{E} S_{n}\left(\alpha_{2}\right) \\
& =-\varepsilon t^{2}-(1+\varepsilon)(c+2 \varepsilon)|t|^{\alpha} L(|t|) \mathbb{E}\left(\sum_{i=1}^{n} R_{i}^{\alpha_{2}}\right) \\
& \leq \log \left(\mathfrak{R e} \mathbb{E} \exp \left(\imath t T_{n}\right)\right) \\
& \leq \varepsilon t^{2}-(1-\varepsilon)(c-2 \varepsilon)|t|^{\alpha} L(|t|) \mathbb{E}\left(\sum_{i=1}^{n} R_{i}^{\alpha_{1}}\right) \\
& =\varepsilon t^{2}-(1-\varepsilon)(c-2 \varepsilon)|t|^{\alpha} L(|t|) \mathbb{E} S_{n}\left(\alpha_{1}\right) .
\end{aligned}
$$

Choose any subsequence $\left\{n_{k}\right\}_{k>1}$ and a right continuous nonincreasing function $\psi$ such that $\varphi_{n_{k}}$ converges to $\bar{\psi}$ at each continuity point of $\psi$, which by Lemma 1 above is all $(1, \infty)$. We see that $\mathbb{E} S_{n_{k}}(\alpha) \rightarrow \psi(\alpha), \mathbb{E} S_{n_{k}}\left(\alpha_{1}\right) \rightarrow \psi\left(\alpha_{1}\right)$ and $\mathbb{E} S_{n_{k}}\left(\alpha_{2}\right) \rightarrow \psi\left(\alpha_{2}\right)$, where necessarily $0<\psi\left(\alpha_{2}\right) \leq \psi(\alpha) \leq \psi\left(\alpha_{1}\right) \leq 1$. We see that for all $|t|$ sufficiently small independent of the subsequence $n_{k} \geq 1$,

$$
\begin{gathered}
-\varepsilon t^{2}-(1+\varepsilon)(c+3 \varepsilon)|t|^{\alpha} L(|t|) \psi\left(\alpha_{2}\right) \leq \log (\mathfrak{R e} \mathbb{E} \exp (\imath t T)) \\
\leq \varepsilon t^{2}-(1-\varepsilon)(c-3 \varepsilon)|t|^{\alpha} L(|t|) \psi\left(\alpha_{1}\right),
\end{gathered}
$$


where $T$ is the nondegenerate limit in (3). Note that if $\psi\left(\alpha_{1}\right)=0$, then because of monotonicity $\psi\left(\alpha_{2}\right)=0$, we would have $\lim _{t \rightarrow 0} t^{-2} \mathbb{E}[1-\cos (t T)]=0$, which by an easy argument based on a classical probability inequality (see Lemma 1, p. 268 of Chow and Teicher [3]), implies that $\mathbb{P}\{T=0\}=1$, contrary to our assumptions. Therefore, $\psi\left(\alpha_{1}\right)>0$.

From (9) we obtain $|t|$ sufficiently small independent of the subsequence $n_{k} \geq 1$,

$$
\begin{aligned}
-\varepsilon-(1+\varepsilon)(c+3 \varepsilon) \psi\left(\alpha_{2}\right) & \leq \log \left(\mathfrak{R e} \mathbb{E} \exp \left(\imath t T_{n_{k}}\right)\right) /\left(|t|^{\alpha} L(|t|)\right) \\
& \leq \varepsilon-(1-\varepsilon)(c-3 \varepsilon) \psi\left(\alpha_{1}\right),
\end{aligned}
$$

where for $\alpha=2$ we use that $\liminf _{t \searrow 0} L(t)>0$; see Remark 1. Since $0<4 \varepsilon<c$ can be made arbitrarily small and $0 \leq \psi\left(\alpha_{1}\right)-\psi\left(\alpha_{2}\right)$ can be made as close to zero as desired, by letting $n_{k} \rightarrow \infty$, we get that for all $|t|$ sufficiently small,

$$
\begin{aligned}
-\varepsilon-(1+\varepsilon)(c+4 \varepsilon) \psi(\alpha) & \leq \log (\mathfrak{R e} \mathbb{E} \exp (\imath t T)) /\left(|t|^{\alpha} L(|t|)\right) \\
& \leq \varepsilon-(1-\varepsilon)(c-4 \varepsilon) \psi(\alpha),
\end{aligned}
$$

which can happen only if $\psi(\alpha)$ does not depend on $\left\{n_{k}\right\}$. Thus (17) holds for some $0<\gamma \leq 1$, namely $\gamma=\psi(\alpha)$.

Proof of Proposition 2. To begin with, we note that whenever (17) holds, necessarily $\mathbb{E} Y=\infty$. To see this, write $D_{n}^{(1)}=\max _{1 \leq i \leq n} Y_{i} /\left(\sum_{i=1}^{n} Y_{i}\right)$ and observe that

$$
\begin{aligned}
\left(D_{n}^{(1)}\right)^{\alpha} & =\max _{1 \leq i \leq n} \frac{Y_{i}^{\alpha}}{\left(\sum_{i=1}^{n} Y_{i}\right)^{\alpha}} \leq S_{n}(\alpha) \\
& \leq \max _{1 \leq i \leq n} \frac{Y_{i}^{\alpha-1}}{\left(\sum_{i=1}^{n} Y_{i}\right)^{\alpha-1}}=\left(D_{n}^{(1)}\right)^{\alpha-1} .
\end{aligned}
$$

From these inequalities it is easy to prove that $\mathbb{E} S_{n}(\alpha) \rightarrow 0, n \rightarrow \infty$, if and only if

$$
D_{n}^{(1)} \rightarrow_{P} 0, n \rightarrow \infty .
$$

Proposition 1 of Breiman [2] says that (10) holds if and only if there exists a sequence of positive constants $B_{n}$ converging to infinity such that

$$
\sum_{i=1}^{n} Y_{i} / B_{n} \rightarrow_{P} 1, n \rightarrow \infty .
$$

Since $\mathbb{E} Y<\infty$ obviously implies (11), it readily follows that $\mathbb{E} S_{n}(\alpha) \rightarrow 0, n \rightarrow \infty$, and thus (7) cannot hold.

We shall first prove the first part of Proposition 2. Following similar steps as in [8] we have that

$$
\begin{aligned}
\mathbb{E} \frac{\sum_{i=1}^{n} Y_{i}^{\alpha}}{\left(\sum_{i=1}^{n} Y_{i}\right)^{\alpha}} & =n \mathbb{E} \frac{Y_{1}^{\alpha}}{\left(\sum_{i=1}^{n} Y_{i}\right)^{\alpha}} \\
& =\frac{n}{\Gamma(\alpha)} \mathbb{E} \int_{0}^{\infty} Y_{1}^{\alpha} e^{-t \sum_{i=1}^{n} Y_{i}} t^{\alpha-1} \mathrm{~d} t \\
& =\frac{n}{\Gamma(\alpha)} \int_{0}^{\infty} t^{\alpha-1} \mathbb{E}\left(e^{-t Y_{1}} Y_{1}^{\alpha}\right)\left(\mathbb{E} e^{-t Y_{1}}\right)^{n-1} \mathrm{~d} t \\
& =: \frac{n}{\Gamma(\alpha)} \int_{0}^{\infty} t^{\alpha-1} \phi_{\alpha}(t) \phi_{0}(t)^{n-1} \mathrm{~d} t .
\end{aligned}
$$


Next, assuming (7) and arguing as in the proof of Theorem 3 in [2] we get

$$
s \int_{0}^{\infty} t^{\alpha-1} \phi_{\alpha}(t) e^{s \log \phi_{0}(t)} \mathrm{d} t \rightarrow \gamma \Gamma(\alpha), \quad s \rightarrow \infty,
$$

where $0<\gamma \leq 1$. For $y \geq 0$, let $q(y)$ denote the inverse of $-\log \varphi_{0}(t)$. Changing the variables to $y=-\log \phi_{0}(t)$ and $t=q(y)$, we get from (12) that

$$
s \int_{0}^{\infty}(q(y))^{\alpha-1} \phi_{\alpha}(q(y)) \exp (-s y) \mathrm{d} q(y) \rightarrow \gamma \Gamma(\alpha), \text { as } s \rightarrow \infty .
$$

By Karamata's Tauberian theorem (see Theorem 1.7.1' on p. 38 of [1]), we conclude that

$$
v^{-1} \int_{0}^{v}(q(x))^{\alpha-1} \phi_{\alpha}(q(x)) \mathrm{d} q(x) \rightarrow \gamma \Gamma(\alpha), \text { as } v \searrow 0
$$

which, in turn, by the change of variable $y=q(x)$ gives

$$
\frac{\int_{0}^{t} y^{\alpha-1} \phi_{\alpha}(y) \mathrm{d} y}{-\log \phi_{0}(t)} \rightarrow \gamma \Gamma(\alpha), \text { as } t \searrow 0 .
$$

Now using that $-\log \phi_{0}(t) \sim 1-\phi_{0}(t)$ as $t \rightarrow 0$, we end up with

$$
\lim _{t \rightarrow 0} \frac{\int_{0}^{t} y^{\alpha-1} \phi_{\alpha}(y) \mathrm{d} y}{1-\phi_{0}(t)}=\gamma \Gamma(\alpha) .
$$

Since $\phi_{\alpha}(y)=\int_{0}^{\infty} e^{-u y} u^{\alpha} G(\mathrm{~d} u)$, by Fubini's theorem

$$
\begin{aligned}
\int_{0}^{t} y^{\alpha-1} \phi_{\alpha}(y) \mathrm{d} y & =\int_{0}^{\infty} u^{\alpha} G(\mathrm{~d} u) \int_{0}^{t} y^{\alpha-1} e^{-u y} \mathrm{~d} y \\
& =\int_{0}^{\infty} G(\mathrm{~d} u) \int_{0}^{u t} z^{\alpha-1} e^{-z} \mathrm{~d} z \\
& =\int_{0}^{\infty} \bar{G}(z / t) z^{\alpha-1} e^{-z} \mathrm{~d} z \\
& =t^{\alpha} \int_{0}^{\infty} \bar{G}(u) u^{\alpha-1} e^{-u t} \mathrm{~d} u
\end{aligned}
$$

A partial integration gives

$$
1-\phi_{0}(t)=t \int_{0}^{\infty} \bar{G}(u) e^{-u t} \mathrm{~d} u
$$

So (13) reads

$$
t^{\alpha-1} \frac{\int_{0}^{\infty} \bar{G}(u) u^{\alpha-1} e^{-u t} \mathrm{~d} u}{\int_{0}^{\infty} \bar{G}(u) e^{-u t} \mathrm{~d} u} \rightarrow \gamma \Gamma(\alpha), \text { as } t \searrow 0,
$$

with $0<\gamma \leq 1$. Let us define the function for $t>0$

$$
f(t)=\int_{0}^{\infty} \bar{G}(u) u^{\alpha-1} e^{-u t} \mathrm{~d} u .
$$

Clearly, $f$ is monotone decreasing and since $\mathbb{E} Y=\infty$, $\lim _{t \rightarrow 0} f(t)=\infty$. We shall show that $f$ is regularly varying at 0 , which by Lemma 3 of Pitman [9, implies that $\bar{G}$ is regularly varying at infinity. We use the identity

$$
u^{1-\alpha} e^{-u t}=\frac{1}{\Gamma(\alpha-1)} \int_{0}^{\infty} y^{\alpha-2} e^{-(y+t) u} \mathrm{~d} y,
$$


which holds for $u>0$ and $\alpha \in(1,2]$. (This is the Weyl-transform, or Weylfractional integral of the function $e^{-u t}$.) This identity combined with Fubini's theorem (everything is nonnegative) gives

$$
\begin{aligned}
\frac{1}{\Gamma(\alpha-1)} \int_{0}^{\infty} y^{\alpha-2} f(y+t) \mathrm{d} y & =\int_{0}^{\infty} \bar{G}(u) u^{\alpha-1} \mathrm{~d} u \frac{1}{\Gamma(\alpha-1)} \int_{0}^{\infty} y^{\alpha-2} e^{-(y+t) u} \mathrm{~d} y \\
& =\int_{0}^{\infty} \bar{G}(u) e^{-u t} \mathrm{~d} u
\end{aligned}
$$

So we can rewrite (14) as

$$
\lim _{t \searrow 0} \frac{t^{\alpha-1} f(t)}{\int_{0}^{\infty} y^{\alpha-2} f(t+y) \mathrm{d} y}=\frac{\gamma \Gamma(\alpha)}{\Gamma(\alpha-1)}=\gamma(\alpha-1) .
$$

A change of variable gives

$$
\int_{0}^{\infty} y^{\alpha-2} f(t+y) \mathrm{d} y=t^{\alpha-1} \int_{1}^{\infty}(u-1)^{\alpha-2} f(u t) \mathrm{d} u,
$$

and so we have

$$
\lim _{t \searrow 0} \int_{1}^{\infty}(u-1)^{\alpha-2} \frac{f(u t)}{f(t)} \mathrm{d} u=[\gamma(\alpha-1)]^{-1} .
$$

We can rewrite $f$ as

$$
f(t)=\int_{0}^{\infty} \bar{G}(u) u^{\alpha-1} e^{-u t} \mathrm{~d} u=t^{-\alpha} \int_{0}^{\infty} \bar{G}(u / t) u^{\alpha-1} e^{-u} \mathrm{~d} u
$$

from which we see that the function

$$
g(t)=\int_{0}^{\infty} \bar{G}(u / t) u^{\alpha-1} e^{-u} \mathrm{~d} u=t^{\alpha} f(t)
$$

is bounded and nondecreasing. Substituting $g$ into (17) we obtain

$$
\lim _{t \rightarrow 0+} \int_{1}^{\infty}(u-1)^{\alpha-2} u^{-\alpha} \frac{g(u t)}{g(t)} \mathrm{d} u=[\gamma(\alpha-1)]^{-1} .
$$

Write $g_{\infty}(x)=g\left(x^{-1}\right), x>0$. Then (18) has the form

$$
\int_{1}^{\infty}(u-1)^{\alpha-2} u^{-\alpha} \frac{g_{\infty}(x / u)}{g_{\infty}(x)} \mathrm{d} u=\frac{k^{M} g_{\infty}(x)}{g_{\infty}(x)} \rightarrow[\gamma(\alpha-1)]^{-1}, \quad \text { as } x \rightarrow \infty
$$

where

$$
k(u)= \begin{cases}(u-1)^{\alpha-2} u^{-\alpha+1}, & u>1 \\ 0, & 0<u \leq 1\end{cases}
$$

and

$$
k^{M} * h(x)=\int_{0}^{\infty} h(x / u) k(u) / u \mathrm{~d} u
$$

is the Mellin-convolution of $h$ and $k$. Note that the Mellin-transform of $k$,

$$
\begin{aligned}
\widetilde{k}(z) & =\int_{1}^{\infty}(u-1)^{\alpha-2} u^{-\alpha-z} \mathrm{~d} u=\int_{0}^{1}(1-v)^{\alpha-2} v^{z} \mathrm{~d} v \\
& =\frac{\Gamma(\alpha-1) \Gamma(1+z)}{\Gamma(\alpha+z)}=\operatorname{Beta}(\alpha-1,1+z),
\end{aligned}
$$

is convergent for $z>-1$. We apply a version of the Drasin-Shea theorem (Theorem 5.2 .3 on p. 273 of [1]). To do this we must verify the following conditions. 
1. $\widetilde{k}$ has a maximal convergent strip $a<\mathfrak{R e} z<b$ such that $a<0$ and $b>0$, $\widetilde{k}(a+)=\infty$ and $\widetilde{k}(b-)=\infty$ if $b<\infty$. Our $\widetilde{k}$ satisfies this condition with $a=-1$ and $b=\infty$.

2. Our function of interest

$$
g_{\infty}(x)=g\left(x^{-1}\right)=\int_{0}^{\infty} \bar{G}(u x) u^{\alpha-1} e^{-u} \mathrm{~d} u, x>0,
$$

is certainly positive and locally bounded.

3. Also our function $g_{\infty}$ is of bounded decrease, since for $\lambda>1$,

$$
\frac{g_{\infty}(\lambda x)}{g_{\infty}(x)}=\lambda^{-\alpha} \frac{(\lambda x)^{\alpha} g(1 /(\lambda x))}{x^{\alpha} g(1 / x)}=\lambda^{-\alpha} \frac{f(1 /(\lambda x))}{f(1 / x)} \geq \lambda^{-\alpha},
$$

so its lower Matuszewska index is at least $-\alpha$.

Therefore, by Theorem 5.2.3 of [1], whenever

$$
\frac{k^{M} g_{\infty}(x)}{g_{\infty}(x)} \rightarrow c, \quad \text { as } x \rightarrow \infty,
$$

then $\widetilde{k}(\rho)=c$ for some $\rho \in(-1, \infty)$. (In our case by (19),$c=[\gamma(\alpha-1)]^{-1}$.) Moreover, since $\widetilde{k}(z)$ is strictly decreasing on $(-1, \infty)$ and $\widetilde{k}(0)=\frac{1}{\alpha-1}$, for any $0<\gamma \leq 1$, the solution $\rho$ to $\widetilde{k}(\rho)=[\gamma(\alpha-1)]^{-1}$ must lie in $(-1,0]$. Theorem 5.2.3 of [1] also says that $g_{\infty}(x)$ is regularly varying at infinity with index $0 \geq \rho>-1$.

Next, since $g_{\infty}(x)=g\left(x^{-1}\right)=x^{-\alpha} f\left(x^{-1}\right) \in \mathcal{R} \mathcal{V}_{\infty}(\rho)$, where $\widetilde{k}(\rho)=c, g \in$ $\mathcal{R} \mathcal{V}_{0}(-\rho)$, which implies that $f \in \mathcal{R} \mathcal{V}_{0}(-\rho-\alpha)$. Recalling that

$$
f(t)=\int_{0}^{\infty} \bar{G}(u) u^{\alpha-1} e^{-u t} \mathrm{~d} u
$$

the Karamata Tauberian theorem now gives that

$$
\int_{0}^{x} \bar{G}(u) u^{\alpha-1} \mathrm{~d} u \in \mathcal{R} \mathcal{V}_{\infty}(\alpha+\rho) .
$$

Thus by Lemma 3 of Pitman $\left[\overline{9}, \bar{G}(u) \in \mathcal{R} \mathcal{V}_{\infty}(\rho)\right.$.

This says that $Y \in D(\beta)$, where $\rho=-\beta \in(-1,0]$ and $\beta$ is the unique solution of

$$
\operatorname{Beta}(\alpha-1,1-\beta)=\frac{\Gamma(\alpha-1) \Gamma(1-\beta)}{\Gamma(\alpha-\beta)}=\frac{1}{\gamma(\alpha-1)} .
$$

We now turn to the proof of the second part of Proposition 2. First consider the case $\beta=0$. Let $0 \leq D_{n}^{(n)} \leq \cdots \leq D_{n}^{(1)}$ denote the order statistics of $Y_{1} /\left(\sum_{i=1}^{n} Y_{i}\right), \ldots, Y_{n} /\left(\sum_{i=1}^{n} Y_{i}\right)$. We see that

$$
\mathbb{E}\left(D_{n}^{(1)}\right)^{\alpha} \leq \mathbb{E} S_{n}(\alpha)=\sum_{i=1}^{n} \mathbb{E}\left(D_{n}^{(i)}\right)^{\alpha} \leq \mathbb{E}\left(D_{n}^{(1)}\right)^{\alpha-1} \leq 1 .
$$

Now $D_{n}^{(1)} \rightarrow_{P} 1$ if and only if $Y \in D(0)$. (See Theorem 1 of Haeusler and Mason [5] and their references.) Thus if $Y \in D(0)$, then (7) holds with $\gamma=1$.

Now assume that $Y \in D(\beta), 0<\beta<1$. In this case, there exists a sequence of positive constants $\left\{a_{n}\right\}_{n \geq 1}$, such that $a_{n}^{-1} \sum_{i=1}^{n} Y_{i} \rightarrow_{d} U$, where $U$ is a $\beta$-stable random variable, with characteristic function

$$
\mathbb{E} e^{\imath t U}=\exp \left\{\beta \int_{0}^{\infty}\left(e^{\imath t u}-1\right) u^{-\beta-1} \mathrm{~d} u\right\} .
$$


Moreover, $Y^{\alpha} \in D(\beta / \alpha)$, and it is easy to check that $a_{n}^{-\alpha} \sum_{i=1}^{n} Y_{i}^{\alpha} \rightarrow_{d} V$, where $V$ is a $\beta / \alpha$-stable random variable, with $\mathrm{cf}$

$$
\mathbb{E} e^{\imath t V}=\exp \left\{\frac{\beta}{\alpha} \int_{0}^{\infty}\left(e^{\imath t u}-1\right) u^{-\beta / \alpha-1} \mathrm{~d} u\right\} .
$$

Since

$$
\begin{aligned}
\lim _{n \rightarrow \infty} n \mathbb{P}\left\{Y>a_{n} u, Y^{\alpha}>a_{n}^{\alpha} v\right\} & =\lim _{n \rightarrow \infty} n \bar{G}\left(a_{n}\left(u \vee v^{1 / \alpha}\right)\right) \\
& =u^{-\beta} \wedge v^{-\beta / \alpha}=: \Pi((u, \infty) \times(v, \infty)),
\end{aligned}
$$

for $u, v \geq 0, u+v>0$, using Corollary 15.16 of Kallenberg [6] one can show that the joint convergence also holds, and the limiting bivariate Lévy measure is $\Pi$. That is,

$$
\left(a_{n}^{-1} \sum_{i=1}^{n} Y_{i}, a_{n}^{-\alpha} \sum_{i=1}^{n} Y_{i}^{\alpha}\right) \rightarrow_{d}(U, V),
$$

where the limiting bivariate random vector has $\mathrm{cf}$

$$
\begin{aligned}
\mathbb{E} e^{\imath(s U+t V)} & =\exp \left\{\int_{[0, \infty)^{2}}\left(e^{\imath(s u+t v)}-1\right) \Pi(\mathrm{d} u, \mathrm{~d} v)\right\} \\
& =\exp \left\{\beta \int_{0}^{\infty}\left(e^{\imath\left(s u+t u^{\alpha}\right)}-1\right) u^{-\beta-1} \mathrm{~d} u\right\} .
\end{aligned}
$$

Since $\mathbb{P}\{U>0\}=\mathbb{P}\{V>0\}=1$, we obtain

$$
S_{n}(\alpha) \rightarrow_{d} \frac{V}{U^{\alpha}}
$$

Thus since $S_{n}(\alpha) \leq 1$ for all $n \geq 1$,

$$
\mathbb{E} S_{n}(\alpha) \rightarrow \mathbb{E}\left(\frac{V}{U^{\alpha}}\right)=: \gamma \leq 1
$$

Clearly $\mathbb{P}\{U<\infty\}=1$, which implies that $0<\mathbb{E}\left(\frac{V}{U^{\alpha}}\right) \leq 1$, and thus by the first part of Proposition 2,

$$
0<\gamma=\frac{\Gamma(\alpha-\beta)}{\Gamma(\alpha) \Gamma(1-\beta)}<1
$$

\section{REFERENCES}

[1] N. H. Bingham, C. M. Goldie, and J. L. Teugels, Regular variation, Encyclopedia of Mathematics and its Applications, vol. 27, Cambridge University Press, Cambridge, 1989. MR:1015093 (90i:26003)

[2] L. Breiman, On some limit theorems similar to the arc-sin law (English, with Russian summary), Teor. Verojatnost. i Primenen. 10 (1965), 351-360. MR0184274 (32 \#1747)

[3] Yuan Shih Chow and Henry Teicher, Probability theory, Independence, interchangeability, martingales, Springer-Verlag, New York-Heidelberg, 1978. MR513230 (80a:60004)

[4] A. Fuks, A. Ioffe, and Dzh. Tolgel's, Expectation of the ratio of the sum of squares to the square of the sum: exact and asymptotic results (Russian, with Russian summary), Teor. Veroyatnost. i Primenen. 46 (2001), no. 2, 297-310, DOI 10.1137/S0040585X97978919; English transl., Theory Probab. Appl. 46 (2003), no. 2, 243-255. MR1968687 (2004b:62045)

[5] Erich Haeusler and David M. Mason, On the asymptotic behavior of sums of order statistics from a distribution with a slowly varying upper tail, Sums, trimmed sums and extremes, Progr. Probab., vol. 23, Birkhäuser Boston, Boston, MA, 1991, pp. 355-376, DOI 10.1007/978-1-46846793-2_12. MR1117277 (92h:60033) 
[6] Olav Kallenberg, Foundations of modern probability, 2nd ed., Probability and its Applications (New York), Springer-Verlag, New York, 2002. MR 1876169 (2002m:60002)

[7] Péter Kevei and David M. Mason, The asymptotic distribution of randomly weighted sums and self-normalized sums, Electron. J. Probab. 17 (2012), no. 46, 21, DOI 10.1214/EJP.v17-2092. MR.2946153

[8] David M. Mason and Joel Zinn, When does a randomly weighted self-normalized sum converge in distribution?, Electron. Comm. Probab. 10 (2005), 70-81 (electronic). MR.2133894 (2005m:60045)

[9] E. J. G. Pitman, On the behavior of the characteristic function of a probability distribution in the neighborhood of the origin, J. Austral. Math. Soc. 8 (1968), 423-443. MR.0231423 (37 \#6978)

Center for Mathematical Sciences, Technische Universität München, BoltzmannSTRASSE 3, 85748 Garching, Germany

E-mail address: peter.kevei@tum.de

Department of Applied Economics and Statistics, University of Delaware, 213 Townsend Hall, Newark, Delaware 19716

E-mail address: davidm@udel.edu 